\title{
Corela
}

Cognition, représentation, langage

HS-20 | 2016

L'Implicite

\section{Impact d'une tâche monologale ou dialogale sur le traitement de l'implicite relatif à l'ethos du locuteur dans un débat}

\section{Bernadeta Wojciechowska}

\section{OpenEdition}

Journals

Édition électronique

URL : http://journals.openedition.org/corela/4700

DOI : $10.4000 /$ corela. 4700

ISSN : 1638-573X

Éditeur

Cercle linguistique du Centre et de l'Ouest - CerLICO

Référence électronique

Bernadeta Wojciechowska, «Impact d'une tâche monologale ou dialogale sur le traitement de l'implicite relatif à l'ethos du locuteur dans un débat », Corela [En ligne], HS-20 | 2016, mis en ligne le 06 décembre 2016, consulté le 20 avril 2019. URL : http://journals.openedition.org/corela/4700 ; DOI 10.4000/corela.4700

Ce document a été généré automatiquement le 20 avril 2019

\section{(c) (1) (2)}

Corela - cognition, représentation, langage est mis à disposition selon les termes de la licence Creative Commons Attribution - Pas d'Utilisation Commerciale - Partage dans les Mêmes Conditions 4.0 International. 


\title{
Impact d'une tâche monologale ou dialogale sur le traitement de l'implicite relatif à l'ethos du locuteur dans un débat
}

\author{
Bernadeta Wojciechowska
}

\section{Introduction}

1 La capacité de compréhension critique des débats polémiques constitue l'un des objectifs majeurs de l'enseignement/apprentissage du français langue étrangère (FLE) au niveau avancé (à partir du niveau B212), et le traitement de l'implicite en fait partie. Le défi pour l'apprenant est de taille puisqu'il engage des connaissances culturelles larges, des capacités intellectuelles de haut niveau (analyse, inférence, comparaison, synthèse) ainsi que des ressources d'attention et de mémoire importantes, afin d'embrasser des interactions argumentatives souvent longues avec l'intervention de plusieurs participants. Nos recherches précédentes, menées en partie en collaboration avec l'équipe de UAM de Poznań et de Université de Rennes $2^{2}$ nous ont permis de prendre conscience de la complexité de l'entreprise et d'identifier certaines difficultés majeures dans des tâches de réponses écrites à des questions préparées par l'enseignant portant sur les débats radiodiffusés, cela même quand les étudiants pouvaient librement gérer le nombre de (ré)écoutes. Parmi les stratégies d'écoute observées, c'est la restitution linéaire des propos entendus accompagnée de l'adoption de la perspective de celui qui parle qui a été considérée par nous comme la moins favorable à l'élaboration d'une compréhension critique du débat. En effet, la restitution linéaire apparait comme une stratégie particulièrement défaillante, notamment par rapport à l'identification et au traitement de l'implicite puisque ceux-ci nécessitent une prise de distance et un dépassement de ce qui est dit à un moment précis du débat afin d'accéder à ce qui est 
présupposé et/ou sous-entendu et de l'envisager dans le contexte plus large du discours et de l'interdiscours.

2 On peut se demander si la forme de questionnaire écrit, rempli individuellement après l'écoute ne participe pas à privilégier cette approche linéaire, et si, par conséquent, d'autres formes pourraient être plus efficaces pour aider l'apprenant à saisir et prendre en compte l'implicite dans l'élaboration de son interprétation du débat. La recherche présentée est basée sur l'hypothèse que la modalité de la discussion à deux - en raison de son mode interactif et négociatif - pourrait constituer une proposition didactique plus adaptée aux objectifs d'identification et d'explicitation des données implicites. Cette hypothèse a été soumise à vérification en confrontant les interprétations des étudiants élaborées dans deux tâches différentes: 1) la réponse individuelle de l'étudiant à des questions fournies par l'enseignant; 2) la discussion en tandem autour des mêmes questions. Nous espérons saisir ainsi l'utilité et les limites de chacune de ces situations didactiques et, de manière plus générale, leur impact sur la qualité de l'interprétation construite.

Dans le présent article, nous nous limitons à l'analyse des productions des apprenants concernant l'ethos d'un expert invité au débat. Nous étudierons dans un premier temps et sur le plan théorique la manière (essentiellement implicite) dont se construit l'ethos dans le discours polémique à travers les dimensions identitaire, subjective et intersubjective. Nous soumettrons ensuite à l'analyse les critères mis en œuvre par les apprenants de FLE lors de la tâche d'interprétation de l'expertise d'un locuteur.

\section{Le débat - à la croisée du discursif, du rhétorique et du didactique}

4 Le guidage en compréhension orale du discours médiatique suppose l'analyse et l'intégration de données venant de disciplines linguistiques diverses et leur transposition sur le terrain de la glottodidactique dans le respect de ses enjeux propres. Nous développons ainsi une réflexion autour de la notion du genre discursif ${ }^{3}$, notion qui suppose et impose l'existence de règles, de scénarios intériorisés auxquels les interlocuteurs se réfèrent plus ou moins consciemment au moment de planifier et d'ajuster leurs actions langagières afin de gérer conjointement la communication.

Le cadre global dans lequel nous plaçons notre réflexion sur la compréhension orale du débat est largement inspiré de travaux en analyse du discours qui définissent le genre comme « un dispositif de communication socio-historiquement définis $»^{4}$ dans lequel les caractéristiques textuelles et formelles sont soumises aux normes sociales propres à un domaine, aux rôles et identités, aux thèmes, aux intentions ainsi qu'à des schémas interactifs spécifiques. ${ }^{5}$

\section{Le débat en tant que genre du discours}

6 Ainsi, le genre débat radiodiffusé pourrait être classé parmi les genres routiniers, selon la conception de D. Maingueneau, puisqu'il appartient au discours médiatique (donc public) et met en scène des invités experts représentant des opinions et des éclairages particuliers sur une question controversée d'intérêt social. Les échanges entre les invités sont gérés et orientés par le journaliste-animateur selon certaines étapes plus ou moins 
stables. C'est à ce journaliste que revient l'introduction de la problématique et la justification de son choix, de sa portée, de ses différents aspects ainsi que la présentation des invités et la légitimation de leur participation dans l'émission (appelé chapeau). C'est lui aussi qui gère la distribution des voix tout au long du débat et sa clôture. Les modes d'interactions et leurs dynamiques sont largement déterminés par cette structure organisatrice et par le type de relations établies entre les différents protagonistes ainsi que par le caractère public du genre. Ainsi, l'interaction se joue à plusieurs niveaux interpersonnels : entre les débatteurs, entre les débatteurs et le journaliste, entre les débatteurs et les auditeurs, entre le journaliste et les auditeurs. Ces négociations sont poursuivies au niveau des rôles et des images respectifs et recoupent les négociations relatives à la gestion des tours de paroles et au contenu argumentatif. Tous ces éléments en se conditionnant mutuellement servent la finalité du débat qui est essentiellement celle de la persuasion et de la formation de l'opinion publique.

7 Autant les éléments énumérés décrivent un modèle abstrait plus ou moins consciemment partagé par les membres d'une communauté discursive et se rapportant à ce que D. Mainguenau appelle la "scène générique", autant sa réalisation concrète appelée "scénario » pourrait être, selon nous, analysée en termes rhétoriques d'ethos, logos, pathos, tels qu'ils sont définis dans le courant de l'argumentation dans le discours. ${ }^{6}$

\section{Le débat en tant que genre rhétorique}

8 Il nous semble possible d'envisager le développement des compétences interprétatives en conjuguant les attentes propres au genre et l'observation des stratégies rhétoriques particulières mises en oeuvre dans une réalisation concrète de celui-ci. La valeur de cette distinction consiste, selon nous, à fournir à l'apprenant d'une part des repères formels et, d'autre part, des outils d'analyse argumentative/rhétorique, tous deux susceptibles d'instruire sa perception et son analyse de différents moments de l'acte interactif. Autrement dit, dans la perspective didactique du développement de compréhension critique du débat radiodiffusé, intégrant la compréhension de l'implicite, on pourrait envisager les dimensions génériques (textuelles, interactives, etc.) comme régies par la visée rhétorique de persuasion et comme ressources instruisant les stratégies de compréhension critique de l'auditeur/apprenant.

Ce choix didactique réduit la portée de l'analyse logique formelle des arguments pourtant dominante dans les pratiques d'enseignement de l'argumentation écrite (genre dissertation). Pour les apprenants, cette analyse de l'écrit est souvent prise comme modèle de rationalité et d'objectivité à l'oral également. Nonobstant, la place ainsi dégagée serait investie par les preuves d'ethos, de pathos et de logos, toutes intégrées. La didactique de la compréhension à l'oral serait alors plus conforme avec les acquis de la recherche sur l'argumentation dans le discours qui démontrent que la force de persuasion ne réside pas dans la valeur logique de l'argument ni même dans la manière idéale de le construire mais qu'elle peut se réaliser à travers différents types de textes ${ }^{7}$ et différentes démarches de persuasion.

\section{L'ethos et son exploitation didactique}

10 Au coeur de notre réflexion sur le modèle didactique du genre « débat radiophonique » se trouve le constat que la formulation des arguments rationnels (logos) est subordonnée à 
une stratégie discursive intégrant d'autres modes d'influence (pathos, ethos), susceptibles de toucher l'auditeur de manière à le séduire, à inspirer sa confiance, tout en discréditant l'opposant. Nous nous focaliserons ici sur l'ethos, et plus particulièrement sur la manière dont les apprenants avancés de FLE intègrent cette dimension dans leur inteprétation du débat.

\subsection{L'ethos et genre de discours}

11 Comme le remarque R. Amossy «toute prise de parole reprend et module un 'ethos préalable'. Le locuteur se fonde sur son statut institutionnel et sur la représentation de sa personne telle que la perçoit selon lui l'auditoire, pour la retravailler en vue de produire une impression appropriée à ses visées argumentatives ${ }^{8}$ ». Cette définition met en valeur la tension entre l'ethos préalable ou prédiscursif (les représentations sociales partagées) et l'ethos actualisé/discursif ainsi que sur son caractère construit et reconstruit en fonction de l'intention du locuteur et des caractéristiques de l'auditoire. L'ancrage de l'ethos dans le système de croyances et de valeurs partagées par une société donnée est évoqué essentiellement sur le mode implicite. Cela place l'apprenant de langue étrangère dans une situation particulièrement délicate puisque l'accès à ces ressources implicites lui est limité.

Il nous paraît utile de suivre le principe édicté par D. Mainguenau ${ }^{9}$ selon lequel le contenu de l'ethos doit être précisé eu égard au genre du discours. Cette remarque a pour nous une valeur didactique supplémentaire en ce qu'elle nous conduit à envisager, dans la pratique d'enseignement/apprentissage, différents types d'ethos en fonction de la spécificité du genre. Il s'agit alors de sensibiliser les apprenants aux endroits dans la structure du genre où l'éthos se manifeste et la fonction qu'il peut y jouer.

\subsection{L'expertise : éthos précisé eu égard au genre débat}

Dans le contexte du débat radiophonique, la notion d'ethos peut être précisée à travers le concept d'enjeu de légitimation « qui vise à déterminer la position d'autorité du sujet ${ }^{10}$ ». En effet, l'expertise présumée des personnes invitées au débat semble un élément clé pour justifier de leur présence et pour donner crédit à leur parole. L'autorité du locuteur peut être validée à travers la revendication de l'autorité institutionnelle ${ }^{11}$ et à sa position sociale qui garantit des savoirs ou des compétences subordonnés à un pouvoir de décision.

14 L'expertise et le pouvoir peuvent se doubler de l'autorité personnelle, fondée sur le pouvoir de séduction du locuteur ainsi que sur sa capacité à communiquer des valeurs morales de politesse, d'ouverture ${ }^{12}$, ou encore à porter la contradiction. Dans le débat, les images discursives affichées par le locuteur sont en plus soumises à l'évaluation et à la négociation de l'interlocuteur ${ }^{13}$ : la valorisation de soi va souvent de pair avec la disqualification de l'autre, parfois dans un jeu très spectaculaire pour ne pas dire violent.

\subsection{L'ethos et expert non engagé}

Dans certains débats polémiques, la scénographie met en scène un invité-expert a priori non engagé - c'est le cas de l'émission exploitée dans notre investigation. Sa présence prend sens dans des débats qui opposent deux parties d'un conflit autour de décisions 
politiques ou de solutions juridiques imminentes. L'expert acquiert dans ce type de configuration le statut d'arbitre, statut qui découle de sa connaissance approfondie du sujet $^{14}$ et de son impartialité présumées. Son arbitrage consiste moins à trancher pour ou contre un argument qu'à l'analyser dans un (des) cadre(s) épistémologique(s) choisi(s). L'objectif est de permettre aux auditeurs de se faire une opinion personnelle nuancée, en quelque sorte par eux-mêmes.

Les attentes des auditeurs face à un expert non engagé porteraient donc essentiellement sur l'éclairage du problème, sur son explication, sur l'explicitation des prémisses implicites des arguments avancés par les parties en conflit, sur les conséquences possibles de chaque choix, etc. Les exigences concerneraient également un niveau élevé de politesse et de culture de débat, corolaire d'une posture rationnelle par opposition aux émotions exprimées par les deux côtés opposés. Sur le plan de la scénographie, la présence d'un expert non engagé aurait donc pour but de rendre le débat rationnel et exhaustif, ce qui ne peut se réaliser qu'à condition d'inviter un expert qui possède les caractéristiques évoquées.

\section{Le compte rendu de la recherche}

La recherche expérimentale dont nous rendons compte ici a été menée en Master 2 dans le cadre d'un cours intitulé « Discours des médias » auprès de 12 étudiantes de philologie romane de niveau $\mathrm{B} 2 / \mathrm{C} 1$. Elle consiste à comparer les démarches d'interprétation et d'évaluation d'un débat radiodiffusé dans deux situations didactiques différentes :

- une situation didactique monologale: il s'agit de répondre individuellement aux questions fournies par l'enseignant par écrit après avoir écouté le débat trois fois avec des pauses pour prendre des notes ;

- une situation didactique dialogale: il s'agit cette fois de discuter à deux autour des questions préparées par l'enseignant dans le but de confronter les observations et les analyses respectives du débat.

L'analyse du chercheur porte ainsi sur les copies écrites des réponses individuelles ainsi que sur l'enregistrement et la transcription des discussions orales. Pour illustrer les tendances observées, nous avons choisi de nous concentrer sur la même question posée dans les deux situations. La question portait sur l'évaluation de l'expertise d'un locuteur. Elle a été formulée comme suit : " Évaluez les réponses de madame Cipriani Crauste sur le succès croissant du tatouage et la signification de ce phénomène. Quels éléments de son discours témoignent en faveur et en défaveur de son expertise?»

Si la question soumise à l'évaluation des apprenants est identique, il faut bien reconnaître que les tâches dans leur ensemble ne sont pas équivalentes, ne serait-ce que du point de vue du nombre d'écoutes possibles ${ }^{15}$. Nous tenons à souligner que notre objectif n'était pas de construire une situation expérimentale classique qui repose sur la constitution de deux groupes : un groupe contrôle et un groupe expérimental, où une variable seulement est manipulée comme c'est l'usage dans les recherches quantitatives. Dans notre investigation, nous avons fait le choix de l'analyse qualitative comme susceptible de saisir les démarches des mêmes personnes dans des situations didactiques différentes. 


\subsection{La présentation du débat soumis à l'interprétation des apprenants}

20 radiophonique Le téléphone sonne du mardi 3 décembre 2013 intitulée « Le succès croissant $\mathrm{du}$ tatouage ${ }^{16} \%$. Le débat s'inscrit dans l'actualité d'un projet de loi interdisant 59 colorants utilisés jusqu'alors pour réaliser des tatouages. Le sujet est introduit par l'animateur Pierre Weill (PW), qui soulève un certain nombre de questions :

«Quels sont les risques de cette pratique? Une soixantaine de colorants devraient être interdits au début de l'année prochaine. Plus généralement, pourquoi cette passion pour le tatouage, notamment chez les jeunes?»

21

Pour débattre, $\mathrm{PW}$ a réuni trois invités : Marie Cipriani-Crauste (MCC), psychosociologue, ex-chercheur au CNRS, non concernée directement par le projet; Jacques Bazex (JB), professeur, membre de l'Académie de médecine et co-auteur d'un rapport en 2007 de l'Académie nationale de médecine sur les complications de la pratique du tatouage, favorable au projet de loi ; et enfin, Tin-tin tatoueur (Tt), président du SNAT (syndicat national des artistes tatoueurs) farouchement opposé au projet de loi.

Les questions posées aux apprenants portent sur l'expertise de MCC dans deux extraits transcrits ci-dessous, qui correspondent aux seuls moments où la parole est donnée à MCC dans l'émission :

$1^{\mathrm{er}}$ extrait

PW : Marie psychosociologue sur ce succès croissant du tatouage, quelle est votre explication?

MCC : de toute façon déjà je vais pas répondre d'entrée parce que le tatouage c'est vraiment disons un phénomène qui est complexe [ouais...] on va pas dire pourquoi et vous n'aurez pas une réponse en fait c'est pas comme ça que ça va se passer en fait tout de même le tatouage existe depuis la nuit des temps...

PW : Oui oui bien sûr

MCC : c'est à-dire que des tatouages il y en a eu toujours, aujourd'hui on peut les montrer, ce qui est différent et donc nous les voyons mais le tatouage s'est toujours fait et dans des conditions peut-être pas très encadrées dans le passé mais est-ce que nous avons comme ça des épidémies et des problèmes énormes?

2ème extrait

PW : Je reviens Marie, cette attirance des jeunes pour le tatouage comment expliquez-vous cela?

MCC : Ecoutez. Je vais vous dire même encore quelque chose. Lorsque j'ai fait mon enquête sur plusieurs années nous sommes allés voir des jeunes c'est-à-dire des mineurs et bien en posant la question ils étaient très très réservés ils pensaient à l'avenir et tous les jeunes ne se laissent pas influencer de cette façon là, bien sûr qu'il y a des jeunes, alors on va en discuter probablement un peu plus tard le pourquoi c'est pas une question qui va se résoudre parce que le tatouage c'est une démarche qui est individuelle, personnelle mais elle a aussi un versant social et à partir de cela on arrive à sortir si vous voulez du problème de la personne c'est le regard de la société et alors là je vous parlerai que justement il y a des pour et des 
contre, les gens qui sont contre vont être probablement des gens qui ne seront pas accessibles aux arguments qui vont être développés par des gens qui sont pour

\subsection{Les catégories d'analyse des données}

- l'identification de l'ethos de MCC et des moyens discursifs mis en œuvre dans sa construction ;

- le traitement interactif de l'ethos de MCC, son inscription dans le débat, son rapport avec les positionnements argumentatifs.

Pour ce qui est de ce premier aspect, nous étions particulièrement sensible au repérage par les étudiants de conduites de MCC visant à construire son image en référence à l'ethos prédiscursif d'intellectuel. À titre illustratif, nous énumérons ici quelques caractéristiques constitutives de cet ethos, en indiquant, de manière sélective, des fragments du discours de CMM où elles semblent activées :

- la priorité donnée à la rationalité, le souci d'objectivité « il y a des pour et des contre les gens qui sont contre»

- le refus de simplification et la recherche de complétude «je vais pas répondre d'entrée parce que le tatouage c'est vraiment disons un phénomène qui est complexe on va pas dire pourquoi et vous n'aurez pas une réponse, en fait c'est pas comme ça que ça va se passer »

- le recours aux recherches et à la communauté scientifique «Lorsque j'ai fait mon enquête sur plusieurs années ", "nous sommes allés voir des jeunes, c'est-à-dire des mineurs »

- le choix du vocabulaire spécialisé, ici celui de l'enquête sociologique « les mineurs », «le versant social ».

Pour ce qui est du traitement interactif de l'ethos revendiqué de MCC, on peut noter son évaluation négative par l'animateur dans le commentaire "oui bien sûr » qui impose l'évidence pour ne pas dire la banalité de l'observation faite par MCC. L'insuffisance de la réponse fournie par la locutrice est signalée implicitement au moment où l'animateur lui donne la parole pour la deuxième fois «Je reviens Marie, cette attirance des jeunes pour le tatouage, comment expliquez-vous cela?». Le temps de parole (moins de deux minutes) de cette invitée dans le débat (qui dure environ 35 minutes) peut être aussi interprété comme évaluation implicite de la qualité faible de son apport.

Quant à MCC elle-même, on remarque sa difficulté à trouver sa place et à jouer pleinement son rôle d'expert impartial et exhaustif. Elle commence sur un ton d'objectivité mais elle s'engage implicitement dans la polémique, prenant position du côté de Tin-tin ( $\mathrm{Tt}$ ) avec un argument qui ne satisfait pas aux standards de rigueur : «le tatouage s'est toujours fait et dans des conditions peut-être pas très encadrées dans le passé mais est-ce que nous avons comme ça des épidémies et des problèmes énormes? ».

Corela, HS-20 | 2016 
Il est assez significatif que Tt ne manque pas de se saisir de ses propos pour les exploiter à ses propres fins.

Sans entrer dans les détails de l'analyse, disons seulement que la confrontation de l'ethos implicitement évoqué et de l'ethos effectivement construit dans le discours autorise pleinement une évaluation négative de l'expertise de MCC. La manière dont elle se déploie s'apparente à vrai dire à une caricature de l'expertise scientifique: la revendication affichée de la valeur scientifique se trouve désavouée simultanément par la faible qualité de l'explication et de l'argumentation. On peut y reprocher une trop grande généralisation et imprécision, l'évocation des faits communément connus ( «le tatouage remonte à la nuit des temps ", " tout le monde peut montrer son tatouage aujourd'hui ", " aujourd'hui on peut les montrer, ce qui est différent et donc nous les voyons ») qui contrastent avec le ton d'une révélation scientifique et par conséquent, donne à la locutrice un air de prétention non justifiée. On peut en conclure que CMM ne satisfait pas aux attentes des auditeurs chevronnés attentes relatives à son ethos implicitement revendiqué de chercheur et de spécialiste dans le domaine de tatouage.

\subsection{La présentation et la discussion des résultats}

Pour la présentation des données, nous avons fait le choix d'analyser les phénomènes récurrents dans les productions des étudiantes à partir d'une transcription complète des réponses monologales et de la réponse dialogale de deux étudiants MP et $\mathrm{AB}$ (en les complétant sélectivement avec quelques autres extraits illustrant les tendances repérées).

\subsubsection{Le traitement de l'expertise en situation monologale}

De manière générale, il faut dire que les réponses écrites ${ }^{17}$ surprennent par leur brièveté, elles comptent en moyenne quatre lignes. La réponse de MP est la plus longue de tout le corpus.

Les réponses écrites individuelles de $\mathrm{MP}$ et $\mathrm{AB}$ sont révélatrices :

MP : Mme Crauste ne veut pas se prononcer trop sur le sujet de succès du tatouage en disant que c'est un phénomène très complexe et en refusant de répondre à la question susmentionnée. Elle ajoute quand même que „maintenant nous voyons les tatouages", alors qu'ils sont plus visibles aujourd'hui et peut-être que nous les cachons plus. Elle ajoute qu'auparavant, malgré les mauvaises conditions, les tatouages ne provoquaient pas d'épidémies. En ce qui me concerne, je ne trouve pas que sa contribution soit importante. Elle est d'accord pour participer à la discussion et après elle refuse de répondre à la question principale de débat [Ecrit.expertise.MP.]

$\mathrm{AB}$ : Le tatouage c'est le phénomène qui existe depuis toujours. C'est un phénomène complexe qui aujourd'hui n'est plus un pratique clandestin, les tatouages peuvent être aujourd'hui montrés dans les rues. M Cipriani Crauste donne certain équilibre dans cette discussion, c'est-à-dire elle montre le phénomène du tatouage en tant qu'une $\mathrm{xxx}$ plutôt $\mathrm{xxx}$ [Ecrit.expertise.A.B]

31 Même si les réponses des deux étudiantes ne présentent pas le même niveau d'analyse, on peut y noter certaines ressemblances. La tendance la plus attestée dans notre corpus d'écrit concerne la posture de restitution ${ }^{18}$ des propos de MCC. Elle se manifeste ainsi dans l'énoncé de MP « Mme Crauste ne veut pas se prononcer trop sur le sujet de succès 
du tatouage en disant que c'est un phénomène très complexe et en refusant de répondre à la question susmentionnée ». Ce caractère de restitution est encore plus visible dans les autres réponses, réalisées essentiellement sur un mode additionnel, à travers le recours au discours indirect avec des phrases introductives telles que " elle dit que ", " elle aussi dit que ", " elle ajoute que ", " elle nie », comme l'illustrent les exemples suivants :

«D'après Marie Cipriani le phénomène du tatouage est plus complexe pour l'expliquer dans quelques mots. Elle aussi dit que les tatouages existent depuis les temps Ancien. Elle nie la thèse de Bazex car elle pretend que la France ne risque l'épidémie des complications après se faire tatouer » [Ecrit.expertise.PS.]

« elle dit que c'est un phénomène très complexe qu'il n'y a pas de réponse adéquate. Elle souligne par contre que les tatouages existent depuis toujours. Mais il y a une différence de ce qui a été autrefois et de ce qui est aujourd'hui » [Ecrit.expertise.MN.]

La restitution se fait parfois aussi sur un mode « objectivant » comme dans cette réponse de $\mathrm{AB}$ qui n'hésite pas à absolutiser les propos de $\mathrm{MCC}$ "C'est un phénomène complexe " [Ecrit.expertise. AB]. Il arrive aussi, souvent, que les étudiantes se réapproprient les propos de MCC : «D'après moi, sa contribution à la discussion montre, que le problème n'est pas nouvel »[Ecrit.expertise.R.]. Les conduites ici observées pourraient être interprétées comme signe de la difficulté des étudiantes à se distancier par rapport au discours entendu.

On peut remarquer la même posture dans les évaluations qui sont au cœur de la question posée aux étudiantes. Dans la plupart des réponses des étudiantes apparaît une évaluation de l'apport de CMM. Ce qui, en revanche, reste plus problématique c'est l'analyse de cet apport et la justification explicite de son évaluation. Ainsi, AB soutient que MCC «donne certain équilibre dans cette discussion » [Ecrit.expertise.AB], on peut penser qu'AB a saisi le rôle communicatif prévu pour CMM. La tentative de justification explicite qui la suit n'aboutit pourtant pas: on n'apprend pas en quoi consiste cet équilibre et à l'appui de quels exemples on peut le justifier. Ce manque d'explicitation des liens entre les propos de MCC et le jugement personnel est caractéristique des réponses fournies dans l'ensemble. Un dernier exemple viendra conforter notre analyse: "Marie Cipriani Crauste reste neutre dans cette discussion, elle explique que le tatouage c'est un phénomène complexe, mais elle n'explique pas pourquoi. Elle dit qu'ils existent toujours, mais aujourd'hui dans la façon différente. Autrefois il y avait les conditions encadrées et elle demande s'il y a aujourd'hui des épidémies » [Ecrit.expertise.D]. Dans cette réponse, on a du mal à saisir un lien implicite entre le jugement de neutralité et la suite de la réponse-

34 A cet égard, la réponse de MP est plus ambigue car on peut se demander si le jugement exprimé n'est pas une sorte de conclusion implicite inférée de la série des propos de CMM citée avant: "En ce qui me concerne, je ne trouve pas que sa contribution soit importante ». Ce qui paraît pourtant plus probable c'est que cette justification est assumée par l'énoncé qui suit : « Elle est d'accord pour participer à la discussion et après elle refuse de répondre à la question principal du débat ». Cette remarque témoigne en tout cas de la capacité de MP à envisager les propos de CMM dans un contexte plus large du débat et de dépasser la linéarité dénoncée précédemment.

En résumé, il apparaît que les réponses à la question sur l'expertise développent paradoxalement très peu cette problématique et privilégient la restitution du contenu explicatif et argumentatif. Cette tendance à privilégier le logos, au détriment de l'ethos, 
apparaît aussi dans les quelques justifications, aussi superficielles et implicites soientelles, des jugements donnés. En effet, les réponses des apprenants, - à l'exception peutêtre de MP - ne tiennent pratiquement jamais compte de la stratégie discursive de MCC, notamment celle relative à son ethos. En tout cas, c'est ainsi que l'on pourrait expliquer le manque d'analyse portant sur l'ethos prédiscursif et sur son actualisation dans le discours par MCC. Il va sans dire que la posture ici décrite ne favorise pas le développement de la compréhension critique du débat.

\subsubsection{Le traitement de l'expertise en situation dialogale}

Nous considérerons les données relatives à la discussion en tandem essentiellement à partir de la transcription de la discussion entre $\mathrm{AB}$ et MP, en la complétant avec quelques extraits de discussions enregistrées d'autres étudiantes.

\begin{tabular}{|c|c|c|}
\hline $\mathrm{AB}$. & 1. & $\begin{array}{l}\text { Eee je dois te dire que j'ai l'impression que j'ai pas très bien compris cette question } \\
\text { (rire) parce que }\end{array}$ \\
\hline & 2. & $\begin{array}{l}\text { si on parle de phénomène de tatouage on peut chercher certains éléments de son } \\
\text { discours qui sont }\end{array}$ \\
\hline & 3. & $\begin{array}{l}\text { pour ou contre les tatouages mais qu'est-ce qu'on peut dire à propos de son } \\
\text { expertise? Selon moi }\end{array}$ \\
\hline & 4. & elle n'est pas assez comment dire \\
\hline MP. & 5. & elle n'est pas nécessaire à la discussion (rire) \\
\hline AB. & 6. & (rire) en général oui \\
\hline MP. & 7. & $\begin{array}{l}\text { parce que si tu invites quelqu'un pour pour faire ee pour faire une discussion et s'il se } \\
\text { prend pour un }\end{array}$ \\
\hline & 8. & $\begin{array}{l}\text { genre de spécialiste et après il refuse de donner la réponse à la question ça n'a pas de } \\
\text { sens parce }\end{array}$ \\
\hline & 9. & $\begin{array}{l}\text { que il le la journaliste la pose la question et elle dit en fait je ne vais pas répondre à } \\
\text { vous ce }\end{array}$ \\
\hline & 10. & n'est pas si simple que ça et voilà (rire) \\
\hline AB. & 11. & donc son expertise n'est pas je ne sais pas pas juste mais eee... \\
\hline MP. & 12. & ...n'existe pas? \\
\hline AB. & 13. & n'existe pas oui (rire) elle hésite beaucoup elle elle dit comme tu as dit \\
\hline MP. & 14. & elle généralise les choses \\
\hline AB. & 15. & Elle généralise les choses elle dit ce n'est pas si simple que ça \\
\hline
\end{tabular}




\begin{tabular}{|l|l|l|}
\hline MP. & 16. & $\begin{array}{l}\text { c'est un phénomène complexe les tatouages existent depuis toujours le tatouage } \\
\text { possède un }\end{array}$ \\
\hline AB. & 18. & $\begin{array}{l}\text { même si elle dit qu'elle a mené ses recherches et elle a fait son expertise a en basant } \\
\text { sur }\end{array}$ \\
\hline & 19 & $\begin{array}{l}\text { ces recherches qui étaient menées depuis depuis longtemps elle dit aussi que elle n'a } \\
\text { pas }\end{array}$ \\
\hline MP. & 21. & Alors comme nous avons déjà dit elle n'était pas nécessaire. \\
\hline
\end{tabular}

[AB./MP.Tatouage-Question4]

La première intervention de $A B$ (lignes 1-4) est emblématique: $A B$ avoue qu'elle ne comprend pas la question. Selon cette étudiante, quand on écoute un débat il faut chercher des arguments pour et contre et non se poser des questions sur l'expertise du locuteur. Sans le savoir, AB confirme notre hypothèse, signalée ci-dessus, sur la posture de l'apprenant privilégiant le logos au détriment de l'ethos. L'observation qui s'impose alors d'entrée de jeu est que la forme dialogale offre la possibilité de négocier le sens de la tâche elle-même, ce qui du point de vue didactique apparaît comme fort précieux. La conséquence immédiate est appréciable : l'interprétation proposée par les étudiantes est plus fine dans le débat en tandem qu'à l'écrit, et s'effectue par étapes.

En effet, en réponse au doute exprimé par $\mathrm{AB}, \mathrm{MP}$ propose sur un ton rieur et un peu provocateur son jugement sur MCC « elle n'est pas nécessaire à la discussion » (ligne 5). $\mathrm{AB}$ l'accepte et MP justifie son évaluation en pointant les aspirations de MCC à être considérée comme une spécialiste. On assiste là à une manifestation de prise de conscience de l'ethos discursif, ce qui constitue une avancée considérable par rapport aux réponses écrites, même si l'ethos en question n'est pas analysé jusqu'au bout. $A B$ collabore avec MP en ajoutant sa conclusion (ligne 11) « donc son expertise n'est pas je ne sais pas pas juste mais eee ». Conclusion à partir de laquelle les deux étudiantes coconstruisent l'évaluation jusqu'à la ligne 15 où la généralisation reprochée par MP est exemplifiée par $\mathrm{AB}$ « elle généralise les choses elle dit « ce n'est pas si simple que ça ».

Justifier ses évaluations est une pratique très présente dans les discussions enregistrées et constitue, à nos yeux, la principale différence entre les deux situations didactiques analysées ici. Avoir un Autre devant et avec soi impose aussi d'argumenter son opinion :

\begin{tabular}{|l|l|l|}
\hline SA. & 18. & $\begin{array}{l}\text { Mais revenons un tout petit peu à ee à cette madame qui parle à ton avis elle } \\
\text { n'apporte pas vraiment }\end{array}$ \\
\hline & 19. & grand elle n'apporte pas grand chose dans la discussion? \\
\hline RB. & 20. & Non \\
\hline
\end{tabular}


\begin{tabular}{|l|l|l|}
\hline SA. & 21. & $\begin{array}{l}\text { moi je suis je pense le contraire parce que je pense qu'elle apaise la discussion trop } \\
\text { émotionnée trop }\end{array}$ \\
\hline
\end{tabular}

\section{SA./RB.Tatouage-Question4}

En plus de la justification des positionnements respectifs, on peut observer aussi que les opinions des apprenants sont prises en charge énonciativement comme c'est le cas deux fois à la ligne 11 «moi, je pense ». On en trouve des occurences encore dans le passage qui suit par exemple à la ligne 20 « pour moi », à la ligne 23 « je pense » :

\begin{tabular}{|c|c|c|}
\hline MN & 9. & $\begin{array}{l}\text { D'accord mais eee mais par contre en ce qui concerne des points faibles d'expertise de } \\
\text { de madame }\end{array}$ \\
\hline & 10. & $\begin{array}{l}\text { Cipriani Crauste son analyse ou au moins les éléments présentés au cours de cette } \\
\text { discussion sont }\end{array}$ \\
\hline & 11. & je dois dire superficiels \\
\hline PS & 12. & Peut-être \\
\hline MN & 13. & $\begin{array}{l}\text { Eee et en vérité elle n'évoque pas aucun argument bien concret et ses constatations } \\
\text { sont eee }\end{array}$ \\
\hline & 14. & $\begin{array}{l}\text { stéréotypées car il est évident qu'il y a des personnes qui sont pour et qui sont contre } \\
\text { les tatouages }\end{array}$ \\
\hline & 15. & $\begin{array}{l}\text { il est aussi clair que le problème de tatouages est complexe mais malheureusement } \\
\text { même si elle est }\end{array}$ \\
\hline & 16. & $\begin{array}{l}\text { un spécialiste et qu'elle a consacré plusieurs années à cette recherche elle n'a pas } \\
\text { arrivé à donner }\end{array}$ \\
\hline & 17. & $\begin{array}{l}\text { nous raison et un simple explication pourquoi les gens vont se faire des tatouages il } \\
\text { n'y a pas cette }\end{array}$ \\
\hline & 18. & $\begin{array}{l}\text { dans dans ces énoncés ces énoncés il n'y a pas il n’y a pas cette réponse e à ee cette } \\
\text { simple }\end{array}$ \\
\hline & 19. & question de base \\
\hline PS & 20. & $\begin{array}{l}\text { Pour moi c'est un avantage qu'elle ne va pas prendre occuper une position claire dans } \\
\text { ce domaine }\end{array}$ \\
\hline & 21. & $\begin{array}{l}\text { car mm le chercheur pour moi doit être plutôt neutre pour effectuer ses recherches } \\
\text { d'une façon eee e }\end{array}$ \\
\hline & 22. & d'une façon claire et objective \\
\hline MN & 23. & $\begin{array}{l}\text { D'accord mais je pense qu'il s'agit plutôt en ce qui concerne cette question } \\
\text { d'explication du }\end{array}$ \\
\hline
\end{tabular}




\begin{tabular}{|l|l|l|}
\hline & 24. & phénomène et pas de donner son opinion \\
\hline PS. & 25. & Peut-être \\
\hline MN & 26. & mais il n'y a pas de cette information \\
\hline & & M./S.Tatouage-Question4 \\
\hline
\end{tabular}

PS/MN.Tatouage-Question4

41 Le passage cité ci-dessus a été choisi pour illustrer la présence des formulations évaluatives, très nombreuses dans les discussions en comparaison avec la situation monologale. Ici aussi l'évaluation de l'expertise s'affine sous l'impulsion de l'Autre, grâce en quelque sorte au positionnement de PS contraire à celui de MN. En effet, MN s'oppose à l'évaluation positive de CMM et, la ligne 11, qualifie ses interventions de «superficielles ». L'hésitation de PS (ligne 12) la pousse à compléter son jugement «en vérité elle n'évoque pas aucun argument bien concret et ses constatations sont stéréotypées » et à donner une suite de preuves introduite par «car» à la ligne 14 . D'autres qualificatifs apparaissent pour dénoncer la banalité des remarques de CMM : «il est évident " (ligne 14), «il est clair » (ligne 15), " pas cette réponse à cette simple question de base » dans les lignes 18-19. MN arrive aussi à contraster les années de recherche brandies par la locutrice avec la qualité de ses réponses, ce qui revient à confronter les données liées à l'ethos revendiqué à celles liées au logos et ce va-et-vient constitue un moment précieux dans le traitement du débat en question.

La suite de cette discussion est aussi pertinente : PS affirme son désaccord, et précise son point de vue (ligne 20) en évoquant l'ethos préalable de chercheur et de sa neutralité. Elle n'arrive pourtant pas à confronter cet ethos avec celui qui est réellement produit. La différence des points de vue conduit MN à la réplique suivante : (ligne 23-24) « d'accord mais je pense qu'il s'agit plutôt en ce qui concerne cette question d'explication du phénomène et pas de donner son opinion ». On assiste alors à un métacommentaire concernant le rôle communicatif de MCC et sa position d'expert non engagé, ce qui témoigne d'une compréhension plus approfondie de la logique découlant de la scénographie mise en place. Aucune occurence de ce type de commentaire n'a été relevée dans les productions écrites.

\section{Conclusion}

Les deux tâches mises à l'épreuve dans le présent article se distinguent par la forme : dialogale $v s$ monologale d'une part et par le nombre d'écoutes auxquelles les apprenants ont eu un accès limité à trois vs. illimité. L'analyse effectuée nous force à penser que la différence décisive pour la qualité de l'évaluation de l'expertise élaborée est celle liée à la présence réelle de l'Autre plus qu'à la possibilité d'écouter l'enregistrement un nombre de fois illimité. C'est cette présence de l'Autre dans la tâche dialogale qui aide à dépasser la posture de restitution dominante dans les tâches écrites. En effet, l'espace créé entre l'Autre et soi favorise la distanciation et la saisie de l'implicite, elle donne lieu à des réponses plus longues, à des jugements plus nombreux et mieux justifiés avec des exemples à l'appui. La forme dialogale semble aussi mieux préserver les apprenants 
contre l'amalgame entre les opinions exprimées par les locuteurs du débat et ses propres opinions.

De plus, la négociation des positionnements respectifs de chacun des auditeurs dans le tandem semble faciliter l'intégration des informations relatives à deux types de preuves en présence : l'ethos (prédiscursif et discursif) et le logos, ce qui aboutit à des évaluations plus fines et plus cohérentes.

L'élaboration commune de l'interprétation critique aide aussi, dans une certaine mesure, une prise de conscience de ses propres conduites d'analyse, y compris à travers des commentaires métacognitifs (p. ex. sur la compréhension de la tâche) ainsi que du genre du débat et de ses réalisations concrètes sous forme de commentaires métacommunicatifs.

Toutefois, il y a un certain nombre de phénomènes qui restent absents des productions des apprenants indépendamment de la tâche. Nous pensons en particulier à l'analyse explicite de l'expertise telle qu'elle est construite implicitement par MCC dans le discours et négociée interactivement par les interlocuteurs, analyse qui serait fondée sur le repérage des marques linguistiques correspondantes. C'est surtout ce dernier aspect, concernant le repérage de marqueurs discursifs de l'implicite, qui nous semble particulièrement important pour l'approfondissement des capacités de compréhension critique. Il permettrait de dépasser les évaluations subjectives en les appuyant sur les données réellement présentes dans les énoncés des interlocuteurs. Les principes généraux de l'approche générique et rhétorique du développement de la compréhension du débat en FLE esquissés succinctement au début du présent article auraient donc besoin d'être précisés pour améliorer la didactique de compréhension orale. ${ }^{19}$

\section{BIBLIOGRAPHIE}

Amossy, R. (2000). L'argumentation dans le discours, Discours politique, littérature d'idées, fiction. Comment peut-on agir sur un public en orientant ses façons de voir, de penser ? Paris : Nathan Université.

Amossy, R. (2010). La présentation de soi. Ethos et identité verbale, Paris : PUF.

Charaudeau, P. (1998). L'argumentation n'est peut-être pas ce que l'on croit. Le français aujourd'hui 123.

Charaudeau, P. (2011). Les médias et l'information, Bruxelles : De Boeck.

Coste, D., North, B., Sheil, J., Trim, J. (2001). Cadre européen commun de références pour les langues. Conseil de l'Europe.

De Chanay H. C. et Kerbrat-Orecchioni, C. (2006). 100 minutes pour convaincre : l'ethos en action de Nicolas Sarkozy. In M. Broth et al. (éd), Le français parlé des médias. Stockholm : Acta Universitatis Stokholmiensis, p. 309-329.

Doury, M. (2003). L'évaluation des arguments dans les discours ordinaires. Langage et société 105, p. 9-37. 
Grize, J.-B. (2004). Le point de vue de la logique naturelle : démontrer, prouver, argumenter. In M. Doury (éd), L'argumentation aujourd'hui. Paris : Presses Sorbonne Nouvelle, p. 35-43.

Górecka, J. et Wojciechowska, B. (2013). Rozwijanie rozumienia publicystycznych audycji radiowych na poziomie zaawansowanym : specyfika etapu poprzedzajĄcego słuchanie. Neofilolog, 40/1, p. 59-78.

Górecka, J. et Wojciechowska, B. (2014). Uwzględnienie procesów współkonstruowania znaczeń w zadaniach słuchania dyskusji radiowej - warunki sukcesu a trudności osób uczĄcych się na poziomie B2/C1. In J. Sujecka-Zajac et al. (éd.), Inspiracja, motywacja,sukces. Rola materiałów dydaktycznych i form pracy na lekcji języka obcego, Warszawa: Wydawnictwo Uniwersytetu Warszawskiego Universitas Varsoviensis, p. 313-326.

Górecka, J., Wilczyńska, W. et Wojciechowska, B. (2015). Developing second language oral competence through an integrated discursive approach: The conceptual framework of the project and the pilot study results. In M. Pawlak et E.Waniek-Klimczak (éd.), Theoretical, empirical and pedagogic perspectives on teaching, learning and assessing speaking skills in a second language. Heidelberg Berlin, Springer-Verlag , p. 29-43.

Kerbrat-Orecchioni, C. (2005). Discours en interaction. Paris : Armand Colin.

Mainguenau, D. (2007). Genres de discours et modes de généricité. Le français aujourd'hui, 159, 29-35.

Richard, E., Wojciechowska, B., Le Bot, M.-C., Wilczyńska, W., Górecka, J., Bourvon, M.-F. (2014). Les débats radiophoniques : quelles stratégies d'écoute pour le niveau avancé ? In : C. Martinot et A. Pegaz Paquet (éd.) Innovations didactiques en français langue étrangère, Paris : CRL éditions, p. 38-50.

Vignaux, G. (2004). Une approche cognitive de l'argumentation. In M. Doury (éd),

L'argumentation aujourd'hui. Paris : Presses Sorbonne Nouvelle, p. 103-124.

Wilczyńska, W. et B. Wojciechowska. (2013). Świadomość gatunku dyskusja a rozumienie obcojęzycznych audycji radiowych. Poznańskie Spotkania JĘzykoznawcze. t. XXVI. Poznań : Wydawnictwo PTPN.

Wojciechowska, B., Górecka, J., Richard, E., Le Bot, M.-C. (2014). Les stratégies rédactionnelles face aux défis d'interprétation d'un débat radiodiffusé. Studia Romanica Posnaniensia XLI/3, p. 117-132.

\section{NOTES}

1. Selon Cadre européen commun de références pour les langues.

2. J. Górecka et B. Wojciechowska (2013, 2014), J. Górecka et B. Wojciechowska (2014), E. Richard et al. (2014), B. Wojciechowska et al. (2014).

3. W. Wilczyńska et B. Wojciechowska (2013), J. Górecka, W. Wilczyńska et B. Wojciechowska (2015).

4. D. Mainguenau (2007: 30$)$.

5. voir par exemple C. Kerbrat-Orecchioni (2005), D. Mainguenau (2007), R. Amossy (2010).

6. Par exemple R. Amossy (2000, 2010), J.-B. Grize (2004), G. Vignaux (2004).

7. Par exemple M. Doury (2003).

8. R. Amossy (2000: 86).

9. D. Maingueneau (2014).

10. P. Charaudeau (1998: 6). 
11. Dans les débats polémiques radiodiffusés français celle de chercheur, journaliste, philosophe, sociologue, responsable d'une organisation.

12. P. Charaudeau (2008).

13. C. Kerbart-Orecchoni (2005), H. C. de Chanay et C. Kerbrat-Orecchioni (2006).

14. Il est souvent chercheur.

15. Trois écoutes, interrompues de temps en temps pour donner le temps de prendre des notes dans la première situation et le nombre d'écoutes illimité dans la deuxième situation. Nous considérons que ce paramètre a un impact limité sur la qualité des interprétations. Au niveau $\mathrm{B} 2 / \mathrm{C} 1$ trois écoutes peuvent être considérées comme largement suffisantes pour saisir les éléments essentiels pour l'élaboration de l'évaluation de l'expertise.

16. http://www.franceinter.fr/emission-le-telephone-sonne-le-succes-croissant-du-tatouage 17. Les réponses des apprenants sont citées ici sans aucune correction de notre part.

18. Les tendances à restitution se répètent dans d'autres corpus dans des situations d'écrit indépendamment du temps alloué à leur exécution, notamment ceux étudiés dans B. Wojciechowska et al. (2014), J. Górecka et B. Wojciechowska $(2013,2014)$.

19. Des éléments allant dans ce sens ont été proposés dans l'article coécrit avec E. Richard soumis à l'acceptation par la rédaction de CORELA.

\section{RÉSUMÉS}

Le présent article aborde la problématique du développement de capacités des étudiants de français langue étrangère à évaluer l'expertise des locuteurs dans un débat radiodiffusé. La recherche présentée se réfère aux travaux menés en analyse du discours et plus particulièrement, à ceux de l'argumentation rhétorique et envisage l'exploitation didactique du concept de l'ethos en glottodidactique. Les modes d'identification et d'interprétation de l'ethos par les étudiants sont analysés dans deux tâches différentes : 1) une tâche monologale (il s'agit d'une réponse individuelle à des questions) et 2) une tâche dialogale (une discussion en tandem). La comparaison des productions des apprenants dans ces deux situations didactiques permet de constater l'impact de la forme de la tâche sur la qualité de l'interprétation de l'implicite relatif à l'ethos.

An impact of a monologue and a dialogue task on the treatment of the speaker's implicit ethos in a radio polemical debate

This research focuses on the development of the French philology students' skills to assess the expertise of the speakers in the radio debate. The proposed study is based on the discourse analysis and the rhetorical argumentation approach and it considers the didactic exploitation of the concept of ethos. The capture and processing of ethos by students are studied in two tasks: 1 ) monologue: an individual response in writing and 2) dialogue: a discussion in pairs. The comparison of students' production in both didactic situations allows to capture their impact on the way of perceiving and clarifying the implicit data concerning ethos. 
INDEX

Mots-clés : compréhension orale en langue étrangère, débat radiodiffusé, interprétation critique, stratégies rhétoriques, ethos, évaluation de l'expertise

Keywords : listening comprehension in a foreign language; radio debate; critical interpretation; rhetoric strategies; ethos; expertise assessment

\section{AUTEUR}

BERNADETA WOJCIECHOWSKA

Université A. Mickiewicz de Poznań, Pologne

bewoj@amu.edu.pl 